\title{
Dual-Channel Electrospray Microchip
}

\author{
Yu Lu, ${ }^{1}$ Fang Liu, ${ }^{1}$ Niels Lion, ${ }^{2}$ Hubert H. Girault ${ }^{1}$ \\ ${ }^{1}$ Laboratoire d'Electrochimie Physique et Analytique, Station 6, Ecole Polytechnique Fédérale de Lausanne, CH-1015 \\ Lausanne, Switzerland \\ ${ }^{2}$ Service Regional Vaudois de Transfusion sanguine, Epalinges, Switzerland
}

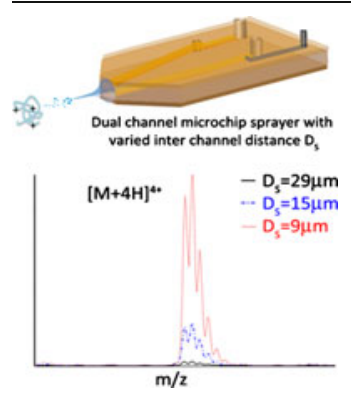

Key words: Dual-channel emitter, Microchip, Micro-electrospray ionization, Mass spectrometry

\begin{abstract}
A dual-channel electrospray microchip has been developed for electrospray ionization mass spectrometry (ESI-MS) where aqueous samples are mixed at the Taylor cone with an organic buffer. Due to the fast and effective mixing in the Taylor cone, the aqueous sample can be well ionized with a high ion intensity. The influence of geometric parameters such as the distance between the two microchannels at their junction at the tip of the emitter has been investigated together with chemical parameters such as the organic buffer composition.
\end{abstract}

Received: 10 August 2012/Revised: 11 November 2012/Accepted: 21 November 2012/Published online: 6 February 2013

\section{Introduction}

E lectrospray ionization (ESI) is one of the most widely used soft ionization techniques for mass spectrometry analysis of biological macromolecules. In the last decade, nano-/micro-electrospray ionization (nano-/micro-ESI) has been developed with small size emitters where the tip diameter ranges from tens to hundreds micrometers so that analytes can be ionized at a low flow rate of below $1 \mu \mathrm{L} / \mathrm{min}[1,2]$. It shows a higher ionization efficiency and ion transmission than the traditional one [3]. With the development of advanced microengineering techniques, our group has pioneered the fabrication of functional electrospray polymer microchips produced by UV-laser photo-ablation and lamination techniques [4-6]. The thinness of these polymer microchips (of the order of hundred micrometers) and the sharpness of the taper shaped emitting tip avoid the wetting by analytes of the edges of the microchips and thus a stable Taylor cone can be generated.

With conventional electrospray emitters, good ionization efficiency requires the addition of organic solvents and volatile acids as a routine procedure [7]. Nevertheless, the specific functions of biological macromolecules can be easily destroyed by organic solvents and acids. To overcome these limitations, nano-electrospray emitters have been used to analyse concentrated aqueous samples even in the absence of organic solvents

Electronic supplementary material The online version of this article (doi:10.1007/s13361-012-0547-z) contains supplementary material, which is available to authorized users.

Correspondence to: Hubert H. Girault; e-mail: hubert.girault@epfl.ch
[8] showing a better ionization performance than the conventional ones. However, the reproducibility and stability of nanoelectrospray still remain a critical issue.

Herein, we utilized a dual-channel microchip fabricated by UV-laser photo-ablation to achieve a stable and efficient electrospray ionization of aqueous peptides. Dual-channel emitters were previously developed in our group for example to study biphasic reactions occurring at the interface of two immiscible phases [6, 9-11]. Here, two miscible solutions of aqueous peptides and of an organic buffer containing an organic solvent and volatile acids were introduced in two microchannels meeting at the tip of the microchip to form a Taylor cone. The quick and thorough mixing in the Taylor cone facilitated the ionization process of aqueous peptides. The influence of different inter-channel separation distances at the tip on the electrospray performance of peptide samples was studied. Additionally, the effects of the composition of the organic buffer on the MS signals of aqueous samples have been investigated.

\section{Experimental}

\section{Chemicals}

A $\beta 16$ (DAEFRHDSGYEVHHQK) was purchased from Bachem (Bubendorf, Switzerland). Acetic acid (HAc) was bought from Sigma-Aldrich (St. Louis, MO, USA). Methanol was purchased from Riedel-de-Haën (Seelze, Germany). Deionized water $(18.2 \mathrm{M} \Omega \cdot \mathrm{cm})$ was obtained from a Milli-Q system from Millipore (Bedford, MA, USA). The lyophilized peptide was dissolved with 
deionized water at a final concentration of $1 \mathrm{mg} / \mathrm{mL}$ as a stock solution stored at $-20{ }^{\circ} \mathrm{C}$ and then was diluted in deionized water before each experiment.

\section{The Fabrication of Dual-Channel Microchip}

The microchannels were ablated by UV-laser excimer LPX 205 (ArF 193 nm) (Lambda Physik, Goettingen, Germany) on a polyimide (PI) film (GoodFellow, Huntingdon, UK). The dualchannel microchip was designed as shown in Figure 1a; one microchannel was fabricated on the upper side of the PI film whilst two microchannels were drilled on the opposite side. This upper microchannel was connected to one of the backside microchannels by a through hole. These connected microchannels were used to supply the organic buffer. The other backside microchannel was used to supply the aqueous sample. The aqueous backside microchannel crosses over the upper organic microchannel thereby defining the tip of the emitter. A black ' $L$ '-shape microchannel was drilled on the backside and filled with carbon ink (Electrador, Electra Polymers Ltd., Roughway Mill, UK). When drilling the backside aqueous microchannel, part of the carbon was exposed thereby defining the electrode to apply the high ESI voltage. The PI microchips with microchannels on both sides were then laminated on both sides with a polyethylene/polyethylene terephthalate composite sheet $(\mathrm{PE} / \mathrm{PET}=25 / 10 \mu \mathrm{m})$ (Payne, Nottingham, UK). The polymer laminate was then cut with a blade under an optical microscope to expose the tip with a taper shape. The separation distance between the two crossing microchannels was varied by varying the depth of the microchannels ranging from ca. $45 \mu \mathrm{m}$ to ca. $55 \mu \mathrm{m}$.

\section{Real-Time Imaging of Micro-Electrospray of Dual-Channel Microchip}

A stainless steel plate counter electrode was placed at a distance of ca. 4-5 mm away from the tip of the dualchannel microchip. A video microscope (Proscope HR [×100 lens], Bodelin Technologies, Lake Oswego, OR, USA) was employed to monitor the electrospray. The experiment was carried out under the same conditions: the organic buffer was composed of $80 \%$ methanol, $2 \%$ $\mathrm{HAc}$, and $18 \%$ water at $12 \mu \mathrm{L} / \mathrm{h}$, whereas the aqueous sample was composed of $5 \mu \mathrm{M} \mathrm{A} \beta 16$ in water at $25 \mu \mathrm{L} /$ $\mathrm{h}$, and a voltage of $3.8 \mathrm{kV}$ was applied at the carbon ink electrode.

\section{Dual-Channel Micro-ESI MS Interface}

The aqueous peptides solution and the organic buffer were loaded in $100 \mu \mathrm{L}$ syringes (Hamilton Bonaduz AG, Bonaduz, Switzerland) and pumped by syringe pumps (KD Scientific, Holliston, MA, USA), respectively. A custom-built holder was used to couple the microchip and the syringe pumps. A LTQ linear ion trap mass spectrometer (Thermo, San Jose, CA, USA) was used in positive ionization mode. The heated capillary was kept at $275^{\circ} \mathrm{C}$. The commercial ESI interface was removed and the holder with microchip was mounted on a plate fixed on the probe slide adapter of the mass spectrometer. The use of high voltage is to be handled cautiously. The current was set between 30 and $70 \mathrm{nA}$ by adjusting the distance between the microchip emitter and the entrance of the MS, and monitored by a nano-ammeter. The ion optics parameters were kept constant for each experiment.

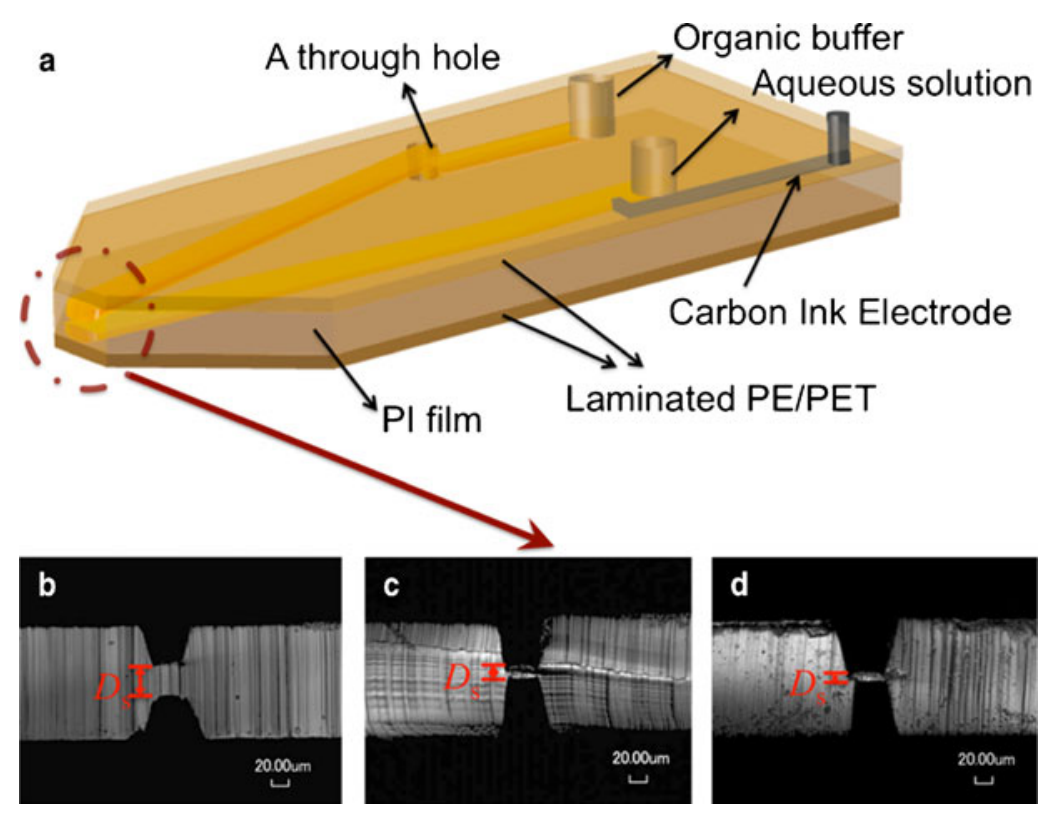

Figure 1. The schematic representation of the dual-channel microchip (a) and the cross section of the tip of dual-channel microchips with different inter-channel separation distances $\left(D_{\mathrm{s}}\right)$ between two microchannels without lamination: (b) $D_{\mathrm{s}}=29 \pm$ $1 \mu \mathrm{m}$; (c) $D_{\mathrm{s}}=15 \pm 1 \mu \mathrm{m}$; (d) $D_{\mathrm{s}}=9 \pm 1 \mu \mathrm{m}$ 
Table 1. The Ion Intensity of Quadruply Charged Target Peptide and Relative Standard Deviation (RSD) of the Total Ion Current Obtained from Dual-Channel Emitters with Different Inter-Channel Separation Distances $\left(D_{\mathrm{s}}\right)$ Between Two Microchannels

\begin{tabular}{ccc}
$\begin{array}{l}\text { The different inter-channel } \\
\text { distance }\left(D_{\mathrm{s}}\right) / \mu \mathrm{m}\end{array}$ & $\begin{array}{c}\text { The ion intensity } \\
\text { of target peptide } * / \times 10^{6}\end{array}$ & $\begin{array}{c}\text { RSD of total } \\
\text { ion current } * / \%\end{array}$ \\
\hline $9 \pm 1$ & 9.81 & 43.7 \\
$15 \pm 1$ & 2.16 & 88.7 \\
$29 \pm 1$ & 0.21 & 114.0 \\
\hline
\end{tabular}

*The mass spectra were recorded under the same experimental conditions: the flow rate of organic buffer $\left(80 \% \mathrm{MeOH}+2 \% \mathrm{HAc}+18 \% \mathrm{H}_{2} \mathrm{O}\right)$ was $12 \mu \mathrm{L} / \mathrm{h}$ and the flow rate of aqueous target peptide $(5 \mu \mathrm{M} \mathrm{A} \beta 16)$ was $25 \mu \mathrm{L} / \mathrm{h} ; 3.8 \mathrm{kV}$ was applied on aqueous phase; distance from the tip to the MS sampling system ca. $4-5 \mathrm{~mm}$

\section{Results and Discussion}

\section{Influence of the Inter-Channel Distance}

Three types of dual-channel microchip with different interchannel separation distances $\left(D_{\mathrm{s}}\right)$ were fabricated and the optical microscope images of the cross section at the tip are shown in Figure $1 \mathrm{~b}-\mathrm{d}$. The $D_{\mathrm{s}}$ values are varied from ca. $29 \mu \mathrm{m}$ to ca. $9 \mu \mathrm{m}$ and accordingly, the depths of microchannels ranged from ca. $45 \mu \mathrm{m}$ to ca. $55 \mu \mathrm{m}$ since the PI substrate has a thickness of $120 \mu \mathrm{m}$.

$A \beta 16$ is a truncated fragment of $A \beta 40 / 42$ peptides, which is involved into Alzheimer's disease. The in vitro reactions of $A \beta$ peptides with reactants such as metal ligands usually are required to mimic physiological conditions (i.e., at $\mathrm{pH}$ values around 7).

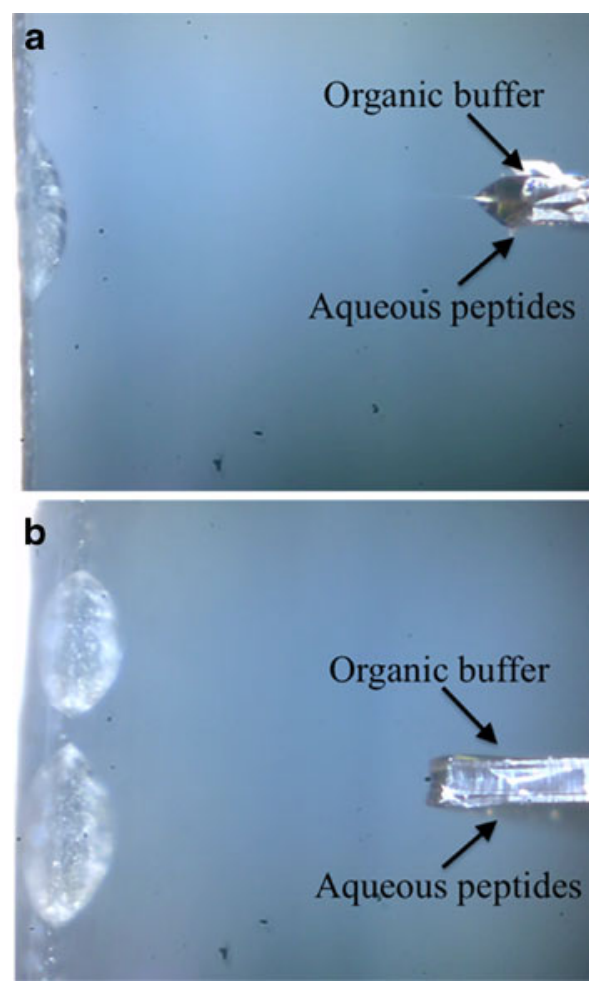

Figure 2. Optical microscopy images of micro-electrospray from dual-channel microchips (a) with $D_{\mathrm{s}}=9 \pm 1 \mu \mathrm{m}$ and (b) with $D_{\mathrm{s}}=29 \pm 1 \mu \mathrm{m}$ under the same experimental conditions
Thus, it was chosen as a target peptide to characterize the performance of the dual-channel microchip. The target peptide was dissolved in pure water in the absence of any organic solvents or acid and then introduced into the microchannel in contact with the carbon electrode, while the organic buffer containing methanol and acetic acid was introduced into the other microchannel. The electrospray from this dual-channel tip was successfully generated when a high voltage was applied. In this way, the organic buffer did not contact the aqueous peptide solution until the Taylor cone formed during electrospray. Methanol and acetic acid inside the organic buffer helped the ionization of aqueous peptides in the Taylor cone likely by decreasing the surface tension of the mixed solution at the tip. As shown in Supplementary Figure S1, triply and quadruply charged target peptides were observed in all three microchips. As $D_{\mathrm{s}}$ value was increased, the ion intensity of charged peptide ions decreased greatly. Moreover, the stability of the total ion current decreased significantly with the increase of $D_{\mathrm{s}}$ values as indicated in Table 1 . The mass spectrometry results were very sensitive on the inter-channel separation distance. On the other hand, the target peptide dissolved in pure water in the absence of any organic solvents or acid also was comparably determined by the commercial ESI source shown in Supplementary Figure S3. The ion intensity of quadruply charged target peptide was lower
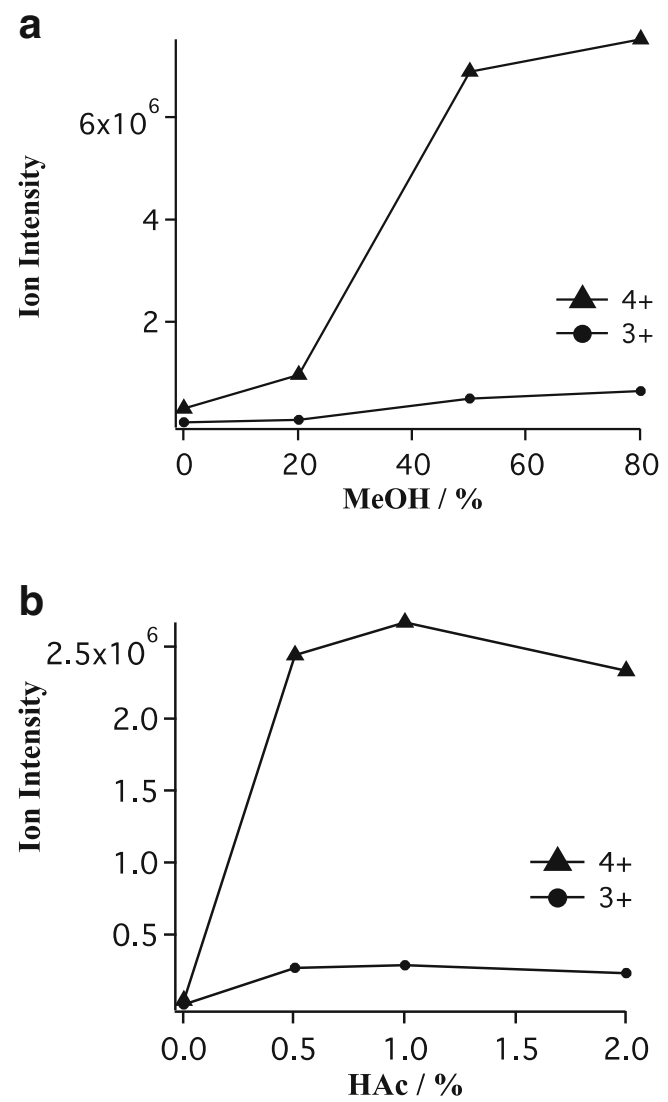

Figure 3. The influences of methanol $(\mathrm{MeOH})(\mathrm{a})$ and acetic acid (HAc) (b) concentration on the ion intensity of $A \beta 16$ peptide under the same experimental conditions with $D_{\mathrm{s}}=9$ $\pm 1 \mu \mathrm{m}$ dual-channel microchip 
than $10^{6}$ magnitude and the RSD of the total ion current was large than 100. The performance is far worse than the dualchannel electrospray microchip.

These mass spectrometry results are just as expected: the cooperative micro-electrospray of two microchannels failed as the separation distance $D_{\mathrm{s}}$ increases. As the $D_{\mathrm{s}}$ values increased to ca. $29 \mu \mathrm{m}$, the ionization efficiency and stability of the target peptide signal decreased to a quality for a single microchannel microchip.

To further confirm this mass spectrometry observation, optical microscopy images of the Taylor cone formed at the dual-channel emitters facing a metallic plate counter electrode have been taken to characterize the electrospray as displayed in Figure 2. The shapes of the Taylor cone turned to be quite different between the two types of dual-channel emitters: the dual-channel emitter with a smaller $D_{\mathrm{s}}$ value (ca. $9 \mu \mathrm{m}$ ) showed only a single well-formed Taylor cone, whereas the emitter with larger $D_{\mathrm{s}}$ value (ca. $29 \mu \mathrm{m}$ ) formed two smaller independent Taylor cones operating simultaneously. Additionally, only one droplet of solutions was accumulated on the counter electrode for the smaller $D_{\mathrm{s}}$ value, whereas two large individual droplets were observed for larger $D_{\mathrm{s}}$ values after several minutes of electrospray. The obtained microscope images demonstrated that the large separation distance destroyed the cooperative electrospray and that two microchannels act as independent single-sprayers despite the fact that the high voltage is only applied to the aqueous microchannel. The coexistence of two Taylor cones may explain the reasons for the extremely low ion intensity of target peptide conducted in large inter-distance microchip as shown in Table 1. Only one electrospray plume containing part of target solution can be directed into the MS inlet and the changeable pathway of electrospray plumes decreases the amount of ionized target peptide able to enter MS inlet. A low percentage of organic buffer has been mixed during the electrospray of aqueous peptides and, thus, the enhancement of ionization also deduced.

Therefore, it has been clearly shown that the smaller the separation distance $D_{\mathrm{s}}$ value, the better the mixing performance of the dual-channel emitter. Considering the limitations of the fabrication technique and the practical manipulation of microchips, the thinnest inter-channel distance that could be achieved was ranging from ca. $5 \mu \mathrm{m}$ to ca. $10 \mu \mathrm{m}$.

\section{Influence of the Organic Buffer Composition}

In order to obtain higher MS signals for aqueous target peptides, the composition of the organic buffer was optimized. Methanol $(\mathrm{MeOH})$ is normally used to decrease the surface tension of analyte samples. The low surface tension of the sample solution facilitates the formation of the Taylor cone and further assists the formation of primary droplets broken from the Taylor cone tip. Acetic acid (HAc) is also added into the analyte solution to enhance the protonation of the analytes in positive ionization mode. Similarly, upon the mixing in the Taylor cone, the concentration of $\mathrm{MeOH}$ and HAc affect the surface tension and $\mathrm{pH}$ value in the Taylor cone and further affect the primary droplets formation. According to Figure 3, as the $\mathrm{MeOH}$ percentage increases, the ion intensity of both quadruply and triply charged $\mathrm{A} \beta 16$ peptides was notably enhanced with the presence of up to $50 \% \mathrm{MeOH}$. Similarly, ion intensity of both quadruply and triply charged $A \beta 16$ peptide was greatly enhanced from $0 \%$ to $0.5 \%$ HAc. Nevertheless, no significant MS signals increase was observed when the proportion of $\mathrm{MeOH}$ exceeds $50 \%$ and that of $\mathrm{HAc}$ $0.5 \%$. Overall, the optimized compositions of both $\mathrm{MeOH}$ and HAc in the organic buffer not only ensure enough high ionization efficiency of aqueous peptides but also increase the performance lifetime of microchip since pure $\mathrm{MeOH}$ can destroy the lamination of microchips to some extent.

As a whole, a rapid and effective mixing of two solutions in the Taylor cone can take place only in millisecond time scale owing to the short diffusion length for two solutions and the large turbulence caused by external electric field. Thus, this dual-channel microchip can successfully enhance the ionization efficiencies of aqueous solutions. Such a phenomenon has been shown to be an efficient tool to supercharge peptides and proteins [12].

\section{Acknowledgments}

The authors thank the China Scholarship Council (CSC) for financial support. The authors also thank Swiss National Science Fundation (SNCF) for the funding for the project of Analytical tools for proteome analysis and redoxomics (grant no 200020_127142/1).

\section{References}

1. Emmett, M.R., Caprioli, R.M.: Micro-electrospray mass-spectrometry ultra-high-sensitivity analysis of peptides and proteins. J. Am. Soc. Mass Spectrom. 5, 605-613 (1994)

2. Wilm, M., Mann, M.: Analytical properties of the nanoelectrospray ion source. Anal. Chem. 68, 1-8 (1996)

3. Gibson, G.T.T., Mugo, S.M., Oleschuk, R.D.: Nanoelectrospray emitters: trends and perspective. Mass Spectrom. Rev. 28, 918-936 (2009)

4. Gobry, V., van Oostrum, J., Martinelli, M., Rohner, T.C., Reymond, F., Rossier, J.S., Girault, H.H.: Microfabricated polymer injector for direct mass spectrometry coupling. Proteomics 2, 405-412 (2002)

5. Lion, N., Gellon, J.O., Jensen, H., Girault, H.H.: On-chip protein sample desalting and preparation for direct coupling with electrospray ionization mass spectrometry. J. Chromatogr. A 1003, 11-19 (2003)

6. Prudent, M., Rossier, J.S., Lion, N., Girault, H.H.: Microfabricated dual sprayer for on-line mass tagging of phosphopeptides. Anal. Chem. 80, 2531-2538 (2008)

7. Cech, N.B., Enke, C.G.: Practical implications of some recent studies in electrospray ionization fundamentals. Mass Spectrom. Rev. 20, 362-387 (2001)

8. Su, S.Q., Gibson, G.T.T., Mugo, S.M., Marecak, D.M., Oleschuk, R.D.: Microstructured photonic fibers as multichannel electrospray emitters. Anal. Chem. 81, 7281-7287 (2009)

9. Mendez, M.A., Prudent, M., Su, B., Girault, H.H.: Peptide-phospholipid complex formation at liquid-liquid interfaces. Anal. Chem. 80, 9499-9507 (2008)

10. Prudent, M., Mendez, M.A., Girault, H.H.: Biphasic electrospray ionization for the study of interfacial complexes. Anal. Sci. 24, 1399-1404 (2008)

11. Prudent, M., Girault, H.H.: Functional electrospray emitters. Analyst 134, 2189-2203 (2009)

12. Miladinovic, S.M., Fornelli, L., Lu, Y., Piech, K.M., Girault, H.H., Tsybin, Y.O.: In-spray supercharging of peptides and proteins in electrospray ionization mass spectrometry. Anal. Chem. 84, 4647-4651 (2012) 